\title{
JOANNA TEKLIK
}

Université Adam Mickiewicz, Poznań

joanna.teklik@amu.edu.pl

\section{EUGÈNE SAVITZKAYA : UNE QUETTE FORMELLE POUR SAVOURER LA VIE}

\begin{abstract}
Teklik Joanna, Eugène Savitzkaya : une quête formelle pour savourer la vie [Eugène Savitzkaya: formal experiments in an effort to make the most of life], Studia Romanica Posnaniensia, Adam Mickiewicz University Press, Poznań, vol. XL/4: 2013, pp. 67-75. ISBN 978-83-232-2635-2. ISSN 01372475. eISSN 2084-4158. DOI: 10.7169/strop2013.404.008.
\end{abstract}

Savitzkaya, hardly known to French public, delights contemporary readers with his style, displaying both peculiar and variegated characteristics. Although he dispenses with the matters of genre, his latest texts (Célébration d'un mariage improbable et illimité, 2002, Exquise Louise, 2003, Fou trop poli, 2005) are still thought to represent the novel. His peculiarity lies in that he contrasts a daily routine with brilliantly worked form, nevertheless well suited to depict it. His predilection for language is pervasive in his style, showing strong preference for details (still, it stands in a stark contrast to the practice of the Nouveaux Romanciers of the 20th century). Savitzkaya asserts himself with the aid of conciseness, draws to a large extent upon surprising stylistic devices, juggles with words, even if paying careful attention to their precision. Finally, he acts as a genuine poet, reviving fragrances and colors of childhood. All in all, the whole of his work is designed to bring his readers to take pleasure in living.

Keywords: Belgium, contemporary novel, Savitzkaya, formal experiments

Les chercheurs s'accordent unanimement sur le plaisir que l'œuvre d'Eugène Savitzkaya procure aujourd'hui au lecteur qui accepte docilement les textes polyphoniques, avec des images suggestives, enfermées dans des phrases riches en anaphores, énumérations et métamorphoses surprenantes. Bien que les formules employées pour la désigner diffèrent, elles évoquent toutes la spécificité de l'écriture savitzkayenne qui, perçue comme « jubilatoire » (Domingues de Almeida), est une constante « célébration de la parole » (Ouellet) et traduit « l' intensité de la vie » (Fabel) et « le bonheur des choses simples » (Bricco) ${ }^{1}$. Or, derrière ledit plaisir, que l'auteur, interviewé à maintes reprises, reconnaît lui-même, se trouve le souci de la forme adéquate. Savitzkaya, sans problématiser les questions qui occupent la société contemporaine, se lance dans une quête formelle indispensable pour présenter la pensée qui se prolonge dans et à travers son écriture. Son parcours littéraire en témoigne indubitablement.

${ }^{1}$ Les mots cités sont empruntés aux titres des articles consacrés à Eugène Savitzkaya. Cf. à ce sujet la bibliographie. 
De mère russe et de père polonais, cet écrivain belge débute à l'âge de dix-sept ans, avec un recueil de poésie qu'il publie à l'occasion du concours Liège des jeunes poètes (Les lieux de la douleur, 1972). D'autres recueils ne se font pas attendre et l'œuvre savitzkayenne se fait remarquer au moment où la Belgique essaie de résoudre ses contradictions communautaires, où l'on s'interroge sur l'avenir de la littérature, en cherchant des auteurs qui puissent concevoir l'écriture en rapport avec l'exigence moderne $^{2}$. Ainsi, tombé en plein débat au sujet de la Belgitude, le jeune Savitzkaya a l'occasion de marquer sa présence : il a sa part dans la publication du numéro culte de La Belgique malgré tout où il évoque ses souvenirs familiaux liés à son enfance (Savitzkaya, 1980 : 425-27). Or, il ne faut pas y voir, comme le souligne judicieusement Marc Quaghebeur, révolte ni proposition formulée autour du futur du pays. La contribution savitzkayenne retrace l'enfance d'un jeune garçon qui évoque les origines slaves de ses parents et raconte leur parcours migratoire jusqu'à Liège, sans chercher vraiment à établir un lien entre son enfance et la nationalité belge ${ }^{3}$. Toutefois, le poids de cet aveu s'avère précieux lorsque l'on constate que les premiers romans savitzkayens s'organisent principalement autour de ces souvenirs ${ }^{4}$ qui peuvent être considérés en l'occurrence comme un point de départ important pour la future mise en fiction ${ }^{5}$.

Savitzkaya-poète se fait petit à petit connaître comme romancier (sans pourtant renoncer à la poésie). Ses textes parus à la fin des années soixante-dix, tels Mentir (1977), Un jeune homme trop gros (1978), La traversée de l'Afrique (1979) sont publiés aux Éditions de Minuit. Ceci n'est pas anodin. Il est d'emblée rangé parmi les auteurs qui, comme l'observe à juste titre Domingues de Almeida ${ }^{6}$, « ne cessent de provoquer, d'une part, le désarroi de la critique et, d'autre part, de susciter une tentative de classement et de division de tendances héritée de la modernité critique et littéraire » (Domingues de Almeida, 2005 : 12). Ainsi, Savitzkaya s'inscrit dans la génération des écrivains belges qui aspirent, tout au long des années quatre-vingts, à l'émergence de la littérature en train de se chercher, en quête de nouvelles voies d'expression. Parallèlement, il est souvent classé par les critiques comme un auteur à part, qui suit sa propre logique et respecte peu les lois du genre. Bien que ses textes narratifs portent tous l'indication générique « roman », ils ressemblent vaguement à des romans, comme l'avoue l'auteur lui-même (Delmez, 1991 : 35). Savitzkaya

${ }^{2}$ Cf. Lettres françaises de Belgique : mutations (1980), Bruxelles : Éditions universitaires/ Archives et Musée de la Littérature, p. 35.

${ }^{3}$ Le désir d'enracinement ne fonde aucune belgitude de son écriture et Savitzkaya, interviewé, n'hésite pas à déclarer ouvertement qu'il ne se sent pas Belge et qu'il vit dans une ville [Liège] plus qu'en Belgique. Cf. Delas, Hordé, 1988 : 120-125.

${ }^{4}$ Les souvenirs familiaux présentent à l'époque des blancs que l'auteur cherche à remplir tout au long de son œuvre, tantôt par le truchement de la fiction, tantôt par le travail de la langue. Cf. à ce sujet Mentir, son roman de 1977, paru aux Éditions de Minuit.

${ }^{5}$ Mentir (1977) et La disparition de maman (1982) sont, à ce titre, révélateurs.

${ }^{6}$ Domingues de Almeida se réfère ici aux propos formulés par F. Schoots, Passer en douce à la douane, L'écriture minimaliste de Minuit, Amsterdam - Atlanta, Rodopi, 1997, p. 12-15. 
reconnaît les conventions normatives du genre tout en les transgressant. Il n'est donc point étonnant que sa place dans la littérature francophone de Belgique reste toujours discutable et son écriture inqualifiable. En 1980 déjà, en témoigne la publication de Terre d'écarts (Miguel, Wouters, 1980), ouvrage où l'on présente et l'on interroge quelques écrivains français de Belgique choisis. L'œuvre de Savitzkaya y figure dans le chapitre intitulé « Le récit et le manque de récit, l'histoire et le manque d'histoire », comme si cette dialectique marquait déjà sa position "à cheval sur deux époques " (cf. Demoulin, 1999). D'une part, les écrits savitzkayens puisent dans la modernité avec, au fond, l'esthétique du Nouveau Roman, à laquelle les jeunes écrivains belges de l'époque, tel, par exemple, J. Ph. Toussaint, s'apparentent ; d'autre part, ils suivent les chemins indiqués par une littérature nouvelle qui ne renonce pas au plaisir du récit ni à l'expression du sujet (Baetens, Viart, $1999: 3$ ).

À cela s'ajoute le fait qu'avec la publication de son premier roman, Mentir, Savitzkaya se situe désormais " à l'intersection du poème et de la prose », (Mertens, 1980 : 74), et il n'est pas le seul d'ailleurs, si l'on prend comme exemple Francis Dannemark, un autre poète passé alors à la prose (Quaghebeur, 1990 : 449). Ce mariage de la poésie et du roman n'est pourtant pas toujours apprécié et certains critiques y voient un déséquilibre, tout en soulevant l'originalité du projet savitzkayen. Ainsi, André Miguel préfère Savitzkaya-romancier à Savitzkaya-poète, car, à le croire, le premier abandonne le récit linéaire, la psychologie et le formalisme du genre, et il emploie une langue dont « la simplicité si sensible et si profonde va beaucoup plus loin que les arabesques trop voyantes ou trop cérébrales ou trop démonstratives de tant d'écritures actuelles » (Miguel, Wouters, 1980 : 70-71). Marc Quaghebeur est plutôt réticent à l'égard de la forme employée par l'auteur de Mentir qui appartient à une autre génération, dit-il, marquée par une obsession rituelle de l'écriture ainsi que par son évolution postmoderne. Cela ne l'empêche pas, pourtant, de considérer les premiers textes de Savitzkaya comme une « chose rare » à la rythmique narrative singulière (Quaghebeur, 1980 : 91) et de voir dans le passage à « un phrasé discursif mais brisé » de la deuxième période de la création savitzkaynenne, un certain prolongement de son travail de réappropriation d'une langue (Quaghebeur, $1990: 429$ ). Maints sont d'ailleurs les chercheurs qui mettent en relief l'exigence moderne propre à l'esthétique savitzkayenne ${ }^{7}$ qui aboutit, aux dires de Patrick Roegiers, à l'invention d'une langue nouvelle, « inouie [...] cacophonique, [...] faite d'assonances et d'allitérations, où le son prime sur le sens » (Quaghebeur, 1980 : 91).

La publication des romans qui suivent, Marin mon coeur (1992) et En vie (1995), ne fait que confirmer ces propos. L'écriture savitzkayenne reste toujours difficilement classable et la poésie s'insinue tellement dans la prose que l'ensemble fait penser à une prose poétique ou bien à « un désordre générique qui se joue des classements d'usage » (Delmez, 1991 : 23). Rien n'est plus fixe et défini de façon catégorique, tout est flou et perd son caractère définitif. Or, la complexité et la richesse des inspi-

${ }^{7}$ Cf. Entretiens avec M. Rombaut, J. Tordeur (1980) in Lettres françaises de Belgique : mutations, Bruxelles, p. 103 et 126. Cf. à ce sujet la thèse de José Domingues de Almeida (Domingues de Almeida, 2005 : 261-263). 
rations savitzkayennes, si variées qu'elles soient, n'excluent pas quelques constantes qui marquent son œuvre poétique et romanesque. Afin de les étudier de près, nous allons nous appuyer sur les écrits les plus récents, à savoir Célébration d'un mariage improbable et illimité (2002), Exquise Louise (2003/2011), Fou trop poli (2005).

Chacun de ces textes reflète la logique optimiste adoptée par l'auteur. Indépendamment de l'histoire racontée, aussi banale et simple qu'elle soit, le lecteur est amené dès le début à fêter, voire célébrer différents événements : le mariage (improbable et illimité) - lorsque « la femme se donne à l'homme, l'homme se donne à la femme, il et elle conjoignent, l'homme et la femme, la femme et l'homme » (Savitzkaya, $2002: 8$ ) ou bien cinquante années de folie qu'un fou se prépare à fêter (Savitzkaya, 2005 : 7), ou encore la conception et la naissance de Louise, qui « grandit dans le jour $[\ldots]$ grandit dans la nuit $[. .]$.$» et qui « naquit avec des yeux sévères qui, grandis-$ sants, s'illuminèrent » (Savitzkaya, 2011, 9-10, 19). Bref, la vie, telle qu'elle est, dans toute sa simplicité et complexité, célébrée dans tous ses états, avec l'inventaire de ses composants les plus petits qui permettent de mieux savourer son goût et son odeur. Et tout ceci à l'aide de l'écriture singulière qui véhicule la réalité dans sa manifestation colorée et fort sensuelle, qui donne au monde une apparence de continuité, qui établit enfin un lien entre l'objet et le corps qui ressent sa présence. Chez Savitzkaya, il n'est point question de communiquer un message au lecteur, mais plutôt d'en faire sentir l'importance, les couleurs et les odeurs, dans un espace chargé de sensualité. Les personnages savtizkayens ne cessent de contempler la beauté du monde, tout en se penchant sur les moindres détails de la nature qui les touchent immuablement. Ainsi, le fou éponyme s'adonne à la contemplation rituelle : « le jour, il admire le soleil qui réchauffe la terre sablonneuse du plateau où il cultive. Et la nuit il admire encore le soleil dont il voit le reflet sur la lune, chaud, mou et agité » (Savitzkaya, $2005: 28$ ) ; la petite Louise, attentive à son milieu naturel, est prise par les choses aussi graves que la capture des escargots dans les herbes, ce qui réclame « une vigilance extrême et une parfaite connaissance du milieu » (Savitzkaya, $2011: 52$ ), enfin un homme parmi les Convives du mariage aussi improbable qu'illimité, secondé par le chœur des autres Convives, médite en observateur le temps qui passe, à travers tous les sens :

[...] je vois l'herbe qui pousse et la nuit qui vient, je vois l'herbe qui fleurit et la nuit qui passe, je vois les étoiles qui s'écoulent goutte à goutte, je vois ce qui est beau et ce qui est laid [...] je sens l'herbe qui pousse je sens le vin qui coule, je sens la lumière qui brille, je sens la bouche de la femme et je sens l'odeur de l'homme, je sens l'odeur du ventre de la femme et je sens l'odeur du nœud de l'homme (pause), j'entends la pluie qui tombe, j'entends l'herbe qui bouge [...] j'entends le vin qui coule et j'entends la femme qui boit, j'entends l'homme et la femme (Savitzkaya, $2002: 36-37)^{8}$.

Ce sont les sens qui structurent le discours du narrateur qui cherche à saisir pleinement l'objet décrit et ce sont les sens qui contribuent à traduire la nature et sa perception par l'homme. Des noces sont célébrées : on boit, on parle, on chante, on jure, on évoque le destin. L'ensemble est un assemblage de sensations qui s'entre-

\footnotetext{
${ }^{8}$ C'est nous qui soulignons.
} 
lacent au rythme quotidien qui marie non seulement le féminin et le masculin, mais aussi l'écriture à la vie ou plutôt la vie à l'écriture. Nous assistons ainsi à une espèce de complémentarité apaisante qui unit de façon éternelle l'homme et la femme, car « ce qui manque à l'une est en l'autre » (Savitzkaya, 2002:20) ${ }^{9}$. La fête continue et accélère par moments, jusqu'à une obsession dont témoignent les questions récurrentes : autour des Convives, le Temps s'exprime par le vrombissement des Mouches, le bourdonnement des Abeilles, le Bruit des Feuilles et des Merlettes et fait ressortir des interrogations fondamentales qui nourrissent tout le roman :

Qui es-tu ? es-tu ? es-tu avec nous ? sommes-nous avec toi ? sommes nous ensemble ? que semblons-nous ? ferons-nous quelque chose ensemble ? que fais-tu de toi ? sommes-nous nés ensemble ? [...] qui sommes-nous ensemble ? que sommes-nous ensemble ? qu'êtes-vous ensemble? devons-nous vivre ensemble ? devez-vous mourir ensemble ? que deviendrons-nous ensemble? (Savitzkaya, $2002: 7$ ).

Il importe que toutes ces questions, comme nous allons le voir plus loin, restent en sursis, donc sans réponse, dans un flux interminable et ne constituent qu'un écho du quotidien.

Sans trop se risquer, on peut avancer la thèse selon laquelle tout texte savitzkayen est imprégné de ladite communion et/ou questionnement ontologique. Il ne cesse de cheminer à travers les méandres de la nature humaine, s'ouvre sans cesse sans pourtant jamais aboutir à une conclusion toute faite. Les mots coulent, tournent et retournent sans qu'il y ait des raisons fondées. Le lecteur est impliqué dans ce vaet-vient constant qui ne lui laisse pas de temps pour la réflexion. Devant ses yeux défilent immuablement des images, des objets, des personnages qui lui échappent, qui se transforment, se succèdent et se répètent. Tout ceci avec un rythme insaisissable, voire obsédant, comme dans le monde de la petite Louise où « Tout est animé, tout est esprit [...] Tout bouge dans tout. Tout se met de concert, avec ou sans harmonie » (Savitzkaya, 2011 : 32), ou bien celui d'un groupe de Convives qui ne cesse de porter des toasts « au monde qui disparaît, au monde qui apparaît, à la femme qui hurle, à l'homme qui gémit, à la femme qui gémit et l'homme qui hurle, à leurs roucoulements de colombe et à leurs cris de coq et pépiements de crapaud » (Savitzkaya, 2002:19). Aussi absurde que cela puisse paraître, l'écriture savitzkayenne se caractérise par un grand souci de composition et l'attention que l'auteur prête au moindre détail, pour, finalement, retracer le chemin de la parole qui se cherche et qui devient, à son tour, son propre objet d'investigation et de questionnement.

Non sans raison, il est légitime de parler en l'occurrence de l'écriture en mouvement ou « en spirale », pour reprendre les mots de Jacques Izoard ${ }^{10}$, tuteur et ami de Savitzkaya. Par ailleurs, l'auteur y souscrit, en déclarant que « vie et écriture doivent être considérées comme un jeu sans fin » (Savitzkaya, 1993 : 193). En effet, son

\footnotetext{
${ }^{9}$ La dualité complémentaire est déjà offerte par le titre même (mariage improbable et illimité) qui évoque l'action statique de l'espace toujours en évolution.

${ }^{10}$ Cf. Prétexte, no 8 , janvier - mars 1995, p. 43. Savitzkaya y explique les circonstances dans lesquelles cette notion fut conçue par Izoard.
} 
œuvre entière s'organise autour de cette idée du mouvement éternel. Le roman, tel que le conçoit Savitzkaya, est un genre informe, libre, ouvert et par là, discontinu. Que ce soit sur le plan formel ou sémantique, la réalité toute entière est inlassablement soumise à un questionnement qui sert d'ouverture et de fermeture à certains textes, comme c'est le cas de Célébration d'un mariage improbable et illimité, ou bien qui structure le récit, de façon quasi naturelle, comme dans les pages d'Exquise Louise. Les questions de la petite fille, du genre « Est-ce que hier c'est déjà demain ?» (Savitzkaya, 2011 : 59) en sont révélatrices et renvoient d'emblée à l'enfance, le temps où l'interrogation nous accompagne de façon naturelle. Et ce ne sont pas vraiment les réponses qui comptent, car l'essentiel réside dans la quête que l'on ne cesse de mener :

Chaque coup de ciseaux de Louise est un signe de renouveau. [...] La résolution est dans la découpe. La solution de toutes les énigmes est dans la découpe. Et toutes les réponses résident dans la découpe. Coupons ce qu'on peut couper sans se fouler le pouce (Savitzkaya, $2011: 46-47)$.

Il en va de même pour le fou éponyme qui, s'interrogeant sur son identité, avoue de ne pas savoir son origine, mais qui, malgré toutes les questions qui « trottent dans sa tête », se satisfait vite d'une auto-réponse, somme toute logique et imprégnée d'une dose d'humour rabelaisien : « [...] je suis bien parti de quelque part et je suis bien sorti de quelqu'un. Je vous assure que ce n'est pas une poule qui m'a pondu ni une jument qui m'a porté dans son ventre [...]» (Savitzkaya, $2005: 13$ ). Le narrateur aborde des questions importantes, tout en détournant l'attention du lecteur qui finit souvent par sourire, indépendamment de la tonalité sérieuse du sujet. C'est ainsi que Savitzkaya glisse parfois certains éléments autobiographiques sans pourtant renoncer à l'exigence formelle. Lorsque le narrateur du Fou trop poli parle de ses racines polono-russes, il soigne plus la mise en forme comme s'il traitait à la légère des choses graves qu'il soulève. Des expressions récurrentes marquent le rythme et le mouvement du récit :

Ceux qui disent que la Russie, dit-il, est blafarde et grise, se trompent lourdement de couleur, car elle n'est pas grise mais bleue, d'un bleu extrêmement délavé et presque blanc qu'un simple petit rayon de soleil ravive. [...] Ceux qui disent que la Pologne est morne et grise, continue-t-il plus loin, se fourrent le doigt dans l'œil, le gauche ou le droit, en tout cas dans celui qui leur sert à mirer les œufs, à mesurer les distances, à faire des clins d'œil, à lorgner les cœurs-de-pigeon ou tout autre chose (Savitzkaya, $2005:$ 13-14).

L'enfance en soi, pour revenir encore un moment à Exquise Louise, bien qu'elle soit un sujet récurrent dans l'œuvre savitzkayenne, n'intéresse l'auteur que pour l'énergie et la ferveur qui lui sont propres et qui sont en mesure de déterminer l'écriture qui en naît. C'est le début de tout ce qui ne peut pas être considéré comme une simple référence, mais devient, d'un texte à l'autre, un topos qui délie les limites d'un lieu déterminé. L'écrivain nous emporte vers le monde, souvent refoulé, de notre enfance, de notre jeunesse, enfin de nos souvenirs, afin de rappeler non seulement les actes inauguraux de notre existence, mais surtout la jouissance vitale et insouciante, gravée dans le quotidien, enracinée dans les gestes simples. Ces sentiments caractérisent, entre autres, la vie du fou savitzkayen qui se compose d'activités banales au 
fond. Tantôt il songe, tantôt il s'adonne à la fête, tantôt il se remet au sport, tantôt il se découvre une vocation d'étudiant, tantôt il contemple la nature, tout en subissant une fascination inouïe (Savitzkaya, $2005: 36$ ). Or, tout ce qu'il fait tient dans ce monde qui est « [...] son alcôve, son alvéole de joie. Sa maison sous les astres » (Savitzkaya, 2005 : 31). Un amour gratuit et innocent pour le monde détermine toute action du héros qui participe à une fête quotidienne. Celle-ci se prolonge sur tous les plans et tourne petit à petit en un absurde, une utopie festive de la folie ordinaire (cf. Scepi, 2013 : sous presse) :

Il festoie toute la journée sacrée, le fou sur son futon. Il festoie avec le plancher. Il festoie avec la théière achetée dans une librairie coranique de Marseille, ô haute pâmoison! Il festoie avec l'espace compris entre les murs, il festoie avec une épluchure de pomme (il a déjà festoyé avec une pomme entière, jeune on a toutes les audaces), il festoie avec la chaise, s'asseyant de côté, en Amazone de cirque (Savitzkaya, 2005 : 38).

À le lire, on croirait se trouver dans un monde voltairien où l'ironie bat son plein et la fameuse technique du projecteur, commentée par Auerbach dans son Mimésis se met en route (Auerbach, 1973). Ne serait-ce que pour faire sourire le lecteur et détourner ses pensées des choses graves ${ }^{11}$. L'image de la célébration, réalisée quotidiennement par le fou, correspond sans doute à celle que nous observons dans Célébration d'un mariage improbable et illimité où, pendant le festin nuptial, des corps s'épousent, s'entrelacent joyeusement, porteurs d'un érotisme et de l'élan vital qui éveille tous les sens.

Il importe de souligner que cette jubilation s'effectue à travers les contradictions, les juxtapositions qui fécondent l'œuvre savitzkayenne, mais aussi les remaniements constants du champ littéraire. Eugène Savitzkaya semble jouer avec la langue, y puise entièrement, tout en recourant à des techniques différentes. Il se plaît à recréer un univers, par les mots et par les voix, pour faire sentir au lecteur son intensité, parfois cruelle et obsédante. En vue de le réaliser, il suspend le temps linéaire de l'action, passe quasi naturellement du quotidien à l'universel, du masculin au féminin, de l'homme à l'animal, à travers tous les âges, à travers toutes les métamorphoses envisageables. Son lexique relève de la transgression et de l'évolution constantes, et les verbes qu'il emploie traduisent le dynamisme du récit et le mouvement circulaire qui caractérise le destin de ses héros. Les derniers écrits de Savitzkaya témoignent toujours de la liberté formelle déclarée par l'auteur. Ils sont souvent fragmentaires, riches en retours ou reprises qui fondent leur spécificité. Chaque partie vaut pour le tout, convoque l'idée d'un ensemble, voire d'une totalité, qu'il appartient au lecteur de composer. Dans ce contexte, les romans savitzkayens devraient se lire comme un assemblage d'idées universelles, exprimées par les sensations et rendues par les images suggestives.

${ }^{11}$ On pense en l'occurrence aux parents de Savitzkaya à qui il rend hommage, une fois de plus, dans Fou trop poli, afin de maintenir le devoir de la filiation, une variante de la continuité formelle qui structure toute son œuvre. La remémoration des membres de la famille absents permet à l'auteur non seulement de combler le vide, mais surtout de faire face à l'avenir. 
Savitzkaya, comme nous l'avons montré plus haut, élabore son chantier, tout en le bousculant, car sa recherche formelle ne finit jamais et elle ne répond qu'à une seule exigence, celle de l'écriture en spirale qui puisse engendrer quelques instants de pure saveur et de jubilation spécifique résultant du (re)maniement des mots, de leurs résonances appariées et de leurs suggestions décantées (Scepi, 2013 : sous presse), comme dans l'exemple ci-dessous :

Ma tisane à moi, est une fleur rouge. La femme descend de la licorne et l'homme descend du singe. Jadis j'étais orang-outan habitant la forêt ancienne, j'avais fui les métropoles et le dur labeur des cités féroces. Redevenu végétarien par joie, j'avais perdu l'usage de la parole. Ma tisane à moi, est une fleur rouge (Savitzkaya, 2005 : 110).

Et pourtant. Cette prose que Savitzkaya met en question et dont il se moque un peu dans « Fou trop poli $»^{12}$, lui sert toujours de cadre officiel et de point de départ important pour sa recherche. À l'image de son fou, l'écrivain belge se plaît à faire évoluer sans cesse son écriture afin de traduire une pensée qu'il prolonge d'un texte à l'autre. Conformément à ses principes, au lieu de parler de la perfection atteinte déjà, il faudrait peut-être parler d'un perfectionnement en cours, d'une quête formelle, annoncée dans le titre. Nouba (2007), le dernier texte visuellement polyphonique de Savitzkaya en est sans doute une expression éloquente.

\section{BIBLIOGRAPHIE}

Baetens, J., Viart, D. (1999): «États du roman contemporain », Écritures contemporaines, n 2, p. 3-7 (introduction).

Delas, D., Hordé, T. (1988): «Entretien avec Eugène Savitzkaya », Le Français d'aujourd'hui, $\mathrm{n}^{\circ} 81$.

Delemez, F. (1991): «Un Autre (de même) », Écritures, nº 1.

Demoulin, L. (1999): «Eugène Savitzkaya à la croisée des chemins », Écritures contemporaines, $\mathrm{n}^{\mathrm{o}} 2$, p. 41-56.

Domingues de Almeida, J. (2005): Auteurs inavoués. Belges inavouables. Fiction, autofiction et fiction de la Belgique dans l'œuvre romanesque de C. Detrez, E. Savitzkaya et J.-Cl. Pirotte. Une triple mitoyenneté. Thèse de doctorat soutenue à l'Université de Porto. http://www. repositorio-aberto.up.pt

Febel, G. (1999): «Écrire l'intensité de la vie ou le roman en excès - l'œuvre romanesque d'Eugène Savitzkaya ", in Les Lettres belges au présent, Actes de congrès des romanistes allemandes, Université d'Osnabrück, 27-29 septembre 1999, p. 281-298.

Mertens, P. (1980): «Entretien », in P. Emond (dir.), Lettres françaises de Belgique : Mutations, Bruxelles : Éditions Universitaires, p. 67-79.

Miguel, A., Wouters, L. (1980): Terre d'écarts. Ecrivains français de Belgique, Bruxelles : Éditions Universitaires.

Ouellet, P. (2004): « La prosopopée exaltée : la célébration de la parole chez Eugène Savitzkaya », Revue des Sciences Humaines, ${ }^{\circ}$ 273, p. 65-81.

12 «Le fou se remet au roman. Et remet ça, par pur plaisir. Qu'est-ce que la prose ? La goutte goutte. Les rats forniquent et sont niqués. Voilà la prose » (Savitzkaya, 2005 : 17). 
Quaghebeur, M. (1980): «Entretien », in P. Emond (dir.), Lettres françaises de Belgique. Mutations, Bruxelles : Éditions Universitaires, p. 81-101.

Quaghebeur, M. (1990): Lettres belges entre absence et magie, Bruxelles : Éditions Labor.

Savitzkaya, E. (1980): «Un jeune Belge », in J. Sojcher (dir.), La Belgique malgré tout, Bruxelles : Éditions de 1'Université de Bruxelles, p. 425-427.

Savitzkaya, E. (1993): Mongolie, Plaine Sale, L'Empire, Rue Obscure, Bruxelles : Éditions Labor.

Savitzkaya, E. (2002): Célébration d'un mariage improbable et illimité, Paris : Les Éditions de Minuit.

Savitzkaya, E. (2003[2011]): Exquise Louise, Paris : Les Éditions de Minuit.

Savitzkaya, E. (2005): Fou trop poli, Paris : Les Éditions de Minuit.

Savitzkaya, E. (2007): Nouba, Crisnée: Yellow Now.

Scepi, H. (2013): «Usages de la folie » (à propos de Fou trop poli d'Eugène Savitzkaya), à paraître in Textyles. Revue des lettres belges de langue française. 